\title{
GRADED RINGS AND KRULL ORDERS
}

\author{
ERIC JESPERS AND PAUL WAUTERS
}

(Communicated by Donald S. Passman)

\begin{abstract}
Let $R$ be a faithfully $S$-graded ring, where $S$ is a submoniod of a torsion-free commutative group and $S$ has no nontrivial units. In case $R$ is a prime Krull order we give necessary and sufficient conditions for $R$ to be a crossed product (respectively a polynomial ring).
\end{abstract}

\section{INTRODUCTION}

We fix some notation and terminology. All rings are associative with unity and all monoids $S$ are torsion-free cancellative commutative, that is all monoids are contained in a torsion-free commutative group. By $e$ we denote the identity of $S$, and $\langle S\rangle$ denotes the group of quotients of $S$ (we use the multiplicative notation). The group of units of $S$ is denoted $U(S)$.

A ring $R$ is said to be $S$-graded, where $S$ is a monoid, if $R=\bigoplus_{s \in S} R_{s}$, a direct sum of Abelian groups, and $R_{s} R_{t} \subseteq R_{s t}$ for all $s, t \in S$. The set $h(R)=\bigcup_{s \in S} R_{s}$ is the set of homogeneous elements of $R$. We denote by $C$ the set of homogeneous elements that are regular in $R$, and $C_{s}=C \cap R_{s}$. We say $R$ is faithfully graded [6] if $\underline{l}\left(R_{s}\right)=\underline{r}\left(R_{s}\right)=0$ for all $s \in S$. Here $\underline{l}\left(R_{s}\right)$ (respectively $\underline{r}\left(R_{s}\right)$ ) denotes the left (respectively right) annihilator of $R_{s}$ in $R$. If, moreover, $S$ is a group and $R_{s} R_{t}=R_{s t}$ then $R$ is said to be strongly graded. Clearly strongly graded rings are faithfully graded. If $A$ is a subset of $S$, then $R_{[A]}=\bigoplus_{a \in A} R_{a}$. A left or right ideal $I$ of $R$ is homogeneous if $I=\bigoplus_{s \in S} R_{s} \cap I$.

Throughout, by the term Goldie ring is meant left and right Goldie ring.

Lemma 1.1. Let $R$ be an $S$-graded ring. If $R$ is a prime Goldie ring such that every essential homogeneous left (or right) ideal of $R$ intersects $C$, then $C$ is a left and right Ore set of $R$.

Proof. We only prove that the multiplicatively closed set $C$ is a left Ore set. Let $r \in R_{t}$ and $c \in C_{s}, s, t \in S$. Then $(R c: r)=\{x \in R \mid x r \in R c\}$ is a

Received by the editors June 9, 1988 and, in revised form, August 16, 1988.

1980 Mathematics Subject Classification (1985 Revision). Primary 16A03, 16A15, 16 A18.

The first named author is supported in part by NSERC-grant OGP0036631. 
homogeneous left ideal of $R$. Since $R$ is a prime left Goldie ring $\left(R c::_{l} r\right)$ is also essential. Hence by the assumption $(R c: r) \cap C \neq \varnothing$, and thus $c^{\prime} r=r^{\prime} c$ for some $c^{\prime} \in C$ and $r^{\prime} \in h(R)$. The left Ore condition for a general $r$ follows easily.

Note that the condition of the lemma holds for P.I.-rings, but in general it is not satisfied (cf. [11]).

If $R$ is an $S$-graded prime Goldie ring such that every essential one-sided ideal intersects $C$, then we denote by $Q^{g}$ the graded classical ring of quotients, i.e., $Q^{g}=C^{-1} R=\left\{c^{-1} r \mid r \in R, c \in C\right\}=\left\{r c^{-1} \mid r \in R, c \in C\right\}=R C^{-1}$. Clearly $Q^{g}$ is a $\langle S\rangle$-graded ring.

Let $R$ be a Krull order in the sense of Chamarie [3, 4], i.e. $R$ is a prime Goldie ring which is a left and right Krull order. It follows that $R$ is a maximal order with the ascending chain condition on integral $c$-ideals. An $R$-ideal $I$ is called a $c$-ideal if $I=(R:(R: I))$, where $(R: I)=\left\{q \in Q_{c l}(R) \mid q I \subset\right.$ $R\}=\left\{q \in Q_{c l}(R) \mid I q \subseteq R\right\}$. By $G(R)$ we denote the free Abelian group of $c$-ideals. Let $\mathbf{P}^{c}(R)$ (respectively, $\mathbf{P}^{n}(R)$ ) be the group all principal $c$ ideals $I$ of $R$ generated by a central (respectively normalizing) element. This means $I=R c=c R$ (respectively $I=R n=n R$ ) where $0 \neq c$ belongs to the quotient field of the center $Z(R)$ of $R$ (respectively $0 \neq n \in Q_{c l}(R)$, the classical ring of quotients of $R$ ). The normalizing (respectively central) class group $C l^{n}(R)$ (respectively $C l^{c}(R)$ ) of $R$ is the quotient group $G(R) / \mathbf{P}^{n}(R)$ (respectively $G(R) / \mathbf{P}^{c}(R)$ ). If moreover $R$ is $S$-graded, then we denote by $C l_{g}^{n}(R)=G_{g}(R) / \mathbf{P}_{g}^{n}(R)$ the graded normalizing class group, and by $C l_{g}^{c}(R)=$ $G_{g}(R) / \mathbf{P}_{g}^{c}(R)$ the graded central class group; where $G_{g}(R)$ is the subgroup of all homogeneous $c$-ideals of $G(R)$, and $\mathbf{P}_{g}^{n}(R)$ and $\mathbf{P}_{g}^{c}(R)$ are defined as above but with $n$ and $c$ homogeneous elements of $Q^{g}$, respectively $Q^{g}(Z(R))$.

In recent years several papers on Krull orders and graded rings have appeared (for example [2, Proposition 5.11; 3, Proposition 3.3; 9, Corollary 4.7; 10, Corollary $2.5 ; 11$, Corollary $3.9 ; 12$, Theorem 3.15 and Corollary 3.17$]$ ). The attention was focused on proving that certain graded rings $R$, such as for example crossed products $R_{e} * S=R_{e}[S, \sigma, y]$ ( $\sigma$ a collection of automorphisms, $\gamma$ a 2-cocycle) over a (semi-) group $S$ with ascending chain condition on cyclic subgroups, are Krull orders whenever $R_{e}$ is a Krull order. Note that a $S$-graded ring $R, S$ a monoid, is called a crossed product if for every $s \in S$ there exists a regular element $\bar{s}$ in $R_{s}$ such that $R_{s}=R_{e} \bar{s}=\bar{s} R_{e}$ and $R_{s} R_{t}=R_{s t}$ for all $s, t \in S$. Or equivalently, $R=R_{e}[S, \sigma, \gamma]$ (the notations and definition are the same as in the group case), where $\sigma$ is a collection of automorphisms of $R_{e}$ and $\gamma$ is a 2-cocycle. In this paper we deal with the converse problem: when is a $S$-graded Krull order $R$ a crossed product? Recently the second author [13] gave an answer in the commutative case if $R$ is a unique factorization domain. In this paper we extend the latter result to the noncommutative case. However our method is completely different from the one used in [13]. 
We will prove the following:

Theorem 1.2. Let $S$ be a torsion-free cancellative commutative monoid with $U(S)=\{e\}$. Let $R$ be a faithfully $S$-graded ring. If $R$ is a Krull order with $C l_{g}^{n}(R)=\{1\}$ then the following are equivalent :

(1) Every essential homogeneous left (or right) ideal of $R$ intersects $C$, and $Q_{e}^{g} \subseteq C_{e}^{-1} R_{e} \cap R_{e} C_{e}^{-1}$

(2) $R \cong R_{e} * S$, a crossed product, and the isomorphism preserves the grading. If any of these conditions holds then $S$ is a free Abelian monoid. If moreover $C l_{g}^{c}(R)=\{1\}$ then $R \cong R_{e}\left[X_{i} \mid i \in I\right]$, a polynomial ring in commuting variables, and $|I|$ is the torsion-free rank of $S$.

The proof of (2) implies (1) is easy. Since $R$ is prime Goldie it follows that $R_{e}$ is semiprime Goldie (see for example [8]). It is then well known (see for example [9]) that $Q_{e}^{g}=Q_{c l}\left(R_{e}\right)=C_{e}^{-1} R_{e}=R_{e} C_{e}^{-1}$.

Note that if $U(S)$ is not trivial then the theorem does not hold anymore. The example on p. 88 in [1] gives a group graded unique factorization domain $R$ satisfying condition 1 , but not condition 2 . Indeed, with notations as in [1], let $R=A_{\mathbf{Q}}=K\left[X_{q} \mid q \in \mathbf{Q}\right]$. Because $\mathbf{Q}$ is a group one easily verifies that condition 1 holds. However if we assume that $R=R_{0} * \mathbf{Q}$, a crossed product, then every $R_{q}, 0 \neq q \in \mathbf{Q}$, contains a unit. However, since $R$ is also a polynomial ring, the only units of $R$ are in $K$ and thus in $R_{0}$; a contradiction. Hence $R$ is not a crossed product. So for general semigroups $S$ we only can apply Theorem 1.2 for the natural $S / U(S)$ gradations (where the quotient semigroup is defined just as in the group case). In case $S=U(S)$ the latter results however have no meaning.

In the remainder of the paper we prove (1) implies (2).

\section{Proof OF THE THEOREM}

Let $R$ be as in the statement of the theorem, and assume condition (1) is satisfied. Hence, by Lemma 1.2, $C$ is a left and right Ore set in $R$. Under these assumptions we obtain

Lemma 2.1. $Q^{g}$ is strongly $\langle S\rangle$-graded with $Q_{[S]}^{g}=C_{e}^{-1} R=R C_{e}^{-1}$. In particular $Q_{[S]}^{g}$ is a left and right localization of $R$.

Proof. Let $s \in S$. Since $\underline{r}\left(R R_{s}\right)=0$ and because $R$ is a prime Goldie ring, $R R_{s}$ is an essential homogeneous left ideal of $R$. So there exists $c \in C_{a s} \cap R_{a} R_{s}$ for some $a \in S$. It follows that $0 \neq c^{-1} R_{a} \subseteq Q_{s^{-1}}^{g}$, and thus $Q_{s^{-1}}^{g} Q_{s}^{g}=Q_{e}^{g}$. Replacing $R R_{s}$ by $R_{s} R, Q_{s}^{g} Q_{s^{-1}}^{g}=Q_{e}^{g}$ follows similarly. Because an arbitrary element $x$ of $\langle S\rangle$ is of the form $s^{-1} t$, for some $s, t \in S$, it is clear that $Q_{x}^{g} Q_{x^{-1}}^{g}=Q_{e}^{g}$. Hence $Q^{g}$ is strongly $\langle S\rangle$-graded.

It is now clear that all regular elements of $R_{e}$ are regular in $Q^{g}$. Thus $C_{e}=\left\{c_{e} \in R_{e} \mid c_{e}\right.$ regular in $\left.R_{e}\right\}$. Hence all elements of $C_{e}$ are invertible in $Q^{g}$ and thus in $Q_{e}^{g}$. Since $\langle S\rangle$ is a torsion-free commutative group it is well known 
that $\langle S\rangle$ can be ordered. Hence it follows from [8] that $Q_{e}^{g}$ is semiprime and hence (cf. [11]) $Q_{e}^{g}$ is a semiprime Goldie ring. Now, as $Q_{e}^{g} \subseteq C_{e}^{-1} R_{e} \cap R_{e} C_{e}^{-1}$ we obtain then that $Q_{e}^{g}$ is its own classical ring of quotients, in particular $Q_{e}^{g}$ has no nontrivial essential one-sided ideals. As $Q_{s^{-1}}^{g} R_{s}$ is such a left ideal we obtain $Q_{s^{-1}}^{g} R_{s}=Q_{e}^{g}$; and similarly $Q_{e}^{g}=R_{s} Q_{s^{-1}}^{g}$ for every $s \in S$. Hence $Q_{s}^{g}=Q_{s}^{g}\left(Q_{s^{-1}}^{g} R_{s}\right)=Q_{e}^{g} R_{s}=C_{e}^{-1} R_{s}$ and similarly $Q_{s}^{g}=R_{s} C_{e}^{-1}$ for every $s \in S$.

The semigroup $S$ is called a Krull semigroup [5] if $S$ is a maximal order (i.e. $(A: A)=\{x \in\langle S\rangle \mid x A \subseteq A\}=S$ for every nonempty ideal $A$ of $S$ ) and $S$ satisfies the ascending chain condition on integral $c$-ideals (also called divisorial ideals). The definition of a $c$-ideal is similar as in the ring case (see [7, p. 215]). Further a $c$-ideal $A$ is called integral if $A \subseteq S$. If moreover $S$ has trivial class group, or equivalently each nonunit of $S$ has a unique factorization into irreducible elements of $S$, then $S$ is called factorial. In [7] it is shown that $S$ is factorial if and only if $S \cong U(S) \times S^{\prime}$ is a direct product, where $S^{\prime}$ is a free Abelian monoid.

Again let $R$ be as in the statement of the theorem, and assume condition (1) is satisfied. We obtain:

Lemma 2.2. $Q_{[S]}^{g}$ is a Krull order with $C l_{g}^{n}\left(Q_{[S]}^{g}\right)=\{1\}$, and $S$ is a factorial monoid.

Proof. Put $B=Q_{[S]}^{g}$. Since $B$ is a left and right localization of $R$ it follows from [3] that, (1) $B$ is a Krull order, and (2) for every $I \in G_{g}(B), I \cap R \in$ $G_{g}(R)$. Hence $C l_{g}^{n}(R)=\{1\}$ implies $C l_{g}^{n}(B)=\{1\}$.

To show that $S$ is a maximal order, let $x \in\langle S\rangle$ and let $A$ be a nonempty ideal of $S$ with ' $x A \subseteq A$. Hence $Q_{x}^{g} B_{[A]} \subseteq B_{[A]}$. As $B_{[A]}$ is a $B$-ideal of the Krull order $B$, we obtain $Q_{x}^{g} \subseteq B$. In particular $x \in S$.

Let $I=\bigoplus_{a \in A} I_{a}, A$ a $S$-ideal (also called fractional ideal) of $S$, be an arbitrary homogeneous $B$-ideal. Since $Q^{g}$ is strongly $\langle S\rangle$-graded with $Q_{[S]}^{g}=$ $B$, and since $Q_{e}^{g}$ has no nontrivial dense ideals, one easily verifies that $(B$ : $I)=Q_{[(S: A)]}^{g}$. Therefore, the integral homogeneous $c$-ideals of $B$ are of the form $B_{[A]}$, where $A$ is an integral $c$-ideal of $S$. Since $B$ is a Krull order it follows that $S$ has the ascending chain condition on integral $c$-ideals. Hence $S$ is a Krull semigroup.

Further if $A$ is an integral $c$-ideal of $S$, then by the previous $B_{[A]}$ is an integral $c$-ideal of $B$. Since $C l_{g}^{n}(B)=\{1\}$ we obtain $B_{[A]}=B n$ for some normalizing homogeneous element $n$ of $B$. Say $n \in Q_{a}^{g}, a \in A$. Hence $B_{[A]}=B_{[S a]}$, and thus $A=S a$. Consequently, $S$ is factorial.

Proof of Theorem 1.2. Since $S$ is factorial and $U(S)=\{e\}, S$ is a free Abelian monoid. Let $\left\{x_{i} \mid i \in I\right\}$ be a free basis for $S$. Clearly $|I|$ is the torsion-free rank of $S$. As before, each $B_{\left[S x_{i}\right]} \in G_{g}(B)$ and thus $B_{\left[S x_{i}\right]} \cap R=R_{\left[S x_{i}\right]} \in G_{g}(R)$. As $C l_{g}^{n}(R)=\{1\}$ this implies $R_{\left[S x_{i}\right]}=R X_{i}$ for some normalizing homogeneous 
element $X_{i} \in R$. Since $U(S)=\{e\}$ and because $X_{i}$ is regular, it follows that $X_{i} \in R_{x_{i}}$ and $R_{x_{i}}=R_{e} X_{i}=X_{i} R_{e}$.

Let $s=x_{1} \cdots x_{n}$ be an arbitrary nontrivial element of $S$. We prove by induction on $n$ that $R_{s}=R_{e} X_{1} \cdots X_{n}$. The case $n=1$ has been shown above. Assume $n>1$. Since $s \in S x_{1}, R_{s} \subseteq R_{\left[S x_{1}\right]}=R X_{1}$. Computing degrees it follows that $R_{s}=R_{x_{2} \cdots x_{n}} X_{1}$. Hence the induction hypothesis yields $R_{s}=R_{e} X_{1} \cdots X_{n}$. Consequently, $R$ is the ring generated by $R_{e} \cup\left\{X_{i} \mid i \in I\right\}$. Since every $X_{i}$ is a normalizing homogeneous element of $R, \sigma_{i}=X_{i}^{-1}-X_{i}$ (i.e. conjugation by $X_{i}$ ) defines an automorphism on $R_{e}$. It is then clear that $R \cong R_{e} * S=R_{e}[S, \sigma]$. This proves (2).

If moreover $C l_{g}^{c}(R)=\{1\}$ then each $X_{i}$ can be taken central. It follows that $X_{i}$ commutes elementwise with $R_{e}$, and $\sigma_{i}$ is the identity mapping. Hence $R=R_{e}\left[X_{i} \mid i \in I\right]$ a polynomial ring in commuting variables.

Remark. In the proof of the theorem we actually did not need that the Krull order $R$ has trivial graded normalizing class group. Essentially all that is needed is that all integral homogeneous $c$-ideals $I$ of $R$ with $P \cap R_{e}=0$ are generated by a normalizing homogeneous element. Or equivalently, every homogeneous height one prime ideal $P$ of $R$ with $P \cap R_{e}=0$ is in $P_{g}^{n}(R)$. Obviously, the latter is satisfied in case condition (2) holds.

\section{REFERENCES}

1. D. F. Anderson, Graded Krull domains, Comm. Algebra 7 (1) (1979), 79-106.

2. D. D. Anderson and D. F. Anderson, Divisibility properties of graded domains, Canad. J. Math. 24 (1982), 196-215.

3. M. Chamarie, Anneaux de Krull non-commutaifs, J. Algebra 72 (1981) 210-222.

4. __ Anneaux de Krull non-commutatifs, Thèse, Université Claude-Bernard (Lyon), 1981.

5. L. G. Chouinard II, Krull semigroups and divisor class groups, Canad. J. Math. 33 (1981), 1459-1468.

6. M. Cohen and S. Montgomery, Group-graded rings, smash products, and group actions, Trans. Amer. Math. Soc. 282 (1), (1984), 237-258.

7. R. Gilmer, Commutative semigroup rings, The University of Chicago Press, Chicago and London, 1984.

8. E. Jespers, On radicals of graded rings and applications to semigroup rings, Comm. Algebra 13 (11) (1985), 2457-2472.

9. E. Jespers and P. Wauters, A general notion of noncommutative Krull rings, J. Algebra 112 (2) (1988), 388-415.

10. H. Marubayashi, Polynomial rings over Krull orders in simple Artinian rings, Hokkaido Math. J. 9 (1980), 63-78.

11. C. Năstăsescu, E. Nauwelaerts, and F. Van Oystaeyen, Arithmetically graded rings revisited, Comm. Algebra 14 (10) (1986), 1991-2017.

12. P. Wauters, $\Omega$-krull rings and Gr- $\Omega$-krull rings in the non-p.i. case, J. Pure Appl. Algebra 32 (1985), 95-113.

13. __ Factorial domains and graded rings, Comm. Algebra 17 (1989), 827-836.

Department of Mathematics, Memorial University of Newfoundland, St. John's, NF, A1C 5S7, CaNADa

Economische Hogeschool Limburg, Universitaire Campus, B-3610 Diepenbeek, Belgium 